\title{
Malignant Neoplasm of the Cervical Esophagus
}

National Cancer Institute

\section{Source}

National Cancer Institute. Malignant Neoplasm of the Cervical Esophagus. NCI

Thesaurus. Code C4763.

A primary or metastatic malignant neoplasm involving the cervical region of the esophagus. 